This manuscript is prepared for Journal of Modern Optics, Feature Issue on the 3rd European Meeting in Physiological Optics, held in the City University, in Sept 2006.

Journal of Modern Optics, Vol. X, No. X, Mmmm 2007, xxx-Xxx

Running heads (verso) Torii et al.

(recto) Dynamic measurement of accommodative responses

\title{
Article
}

\section{Dynamic measurement of accommodative responses while viewing stereoscopic images}

MASAHITO TORII $\dagger$, YUUKI OKADA $\dagger$, KAZUHIKO UKAI*†, JAMES S.

WOLFFSOHN $\ddagger$ AND BERNARD GILMARTIN $\ddagger$

† School of Science and Engineering, Waseda University, Shinjuku, Tokyo 169-8555, Japan

$\ddagger$ Ophthalmic Research Group, School of Life and Health Sciences, Aston University, Birmingham B4 7ET, UK

*Correspondence Author: Kazuhiko Ukai. Email: ukai@waseda.jp 


\begin{abstract}
Using video refraction accommodative and convergence dynamic responses were measured to stepped changes in convergence stimuli with unchanged accommodative stimuli (conflicting stereoscopic image) and compared with responses to nonconflicting target stimuli. Three targets were used that varied in their spatial frequency components. An accommodative transient overshoot was evident in 4 out of 7 subjects for only conflicting stimuli. One showed accommodative and convergence oscillation probably due to difficulty in fusing the stereoscopic target when it had a higher spatial component, however, this oscillation diminished when the target was spatial low-pass filtered. We hypothesize that transient responses to step stimuli is initiated by convergence-driven accommodation and subsequently followed by slower fine-control of accommodation modulated by the amount of blur. Inter-subject differences in convergence-driven accommodation may also be a factor to consider. For stereoscopic stimuli, it is proposed that the increase in blur immediately after the onset of the accommodative response inhibits cessation of the response. (156 words)
\end{abstract}

Keywords: accommodation; convergence; blur; spatial frequency; stereoscopic display; visual fatigue 


\section{Introduction}

Asthenopia is a frequent complaint of viewers of stereoscopic images. It has been suggested that asthenopia is caused when there occurs a conflict between the accommodative and convergence stimuli [1].

Okada et al. [2] measured accommodative and convergence responses to convergence stimuli varied in a step-wise manner on a stereoscopic liquid crystal display (LCD). The display had a parallax barrier which generated 2 images presented to the two eyes separately. Accommodative responses of one eye were measured when accommodation and convergence were in conflict using a modified automatedrefractometer and static responses were assessed when dynamic responses had stabilised. Convergence responses of the contralateral eye were simultaneously measured using a limbus tracking method. Stimulus presentation comprised two conditions: conflict between accommodation and convergence (stereoscopic mode) and a non-conflicting natural condition (non-stereoscopic mode). Three targets were generated each of which differed with regard to spatial components. The Maltese cross target which initially possessed higher spatial frequency components was blurred by calculation of convolution with 2 Gaussian blurs which are equivalent to low-pass filters.

It was found that the conflict between accommodative stimuli and convergence stimuli in stereoscopic display affects static accommodative responses, that is, accommodative responses were most closely matched to the screen position when the target had higher spatial components, but most closely matched the convergence stimulus when the spatial components of the target were filtered. As lower spatial frequency components of the targets are tolerant to defocus, defocus-driven accommodation to the target was weak without higher spatial components and hence convergence-driven accommodation was predominant. Therefore the balance between blur-driven accommodation and convergence-driven accommodation to a stereoscopic display is a function of spatial frequency components of the target.

Furthermore, in some subjects dynamic accommodative responses were recorded and showed a large accommodative overshoot when the target had high spatial frequency components. The overshoot was not observed in convergence recordings. It was found in all subjects that the convergence-driven accommodation was greater and more dominant depending on the degree of blur. Dynamic responses could only be 
measured in a few subjects owing to the difficulty in aligning the refractometer during convergence eye movements.

Modeling of the accommodation and convergence control system suggests that accommodative step responses consist of 2 phases: a fast component mediated by convergence and a sustained component mediated by defocus. Accommodative overshoot is evident when a conflict between accommodative stimulus and convergence stimulus occurs because the sustained component following the fast component is diminished. This accommodative overshoot became larger as the blur demands of the target increased, and can be used to quantify the challenge to dynamic accommodative response where there is conflict between accommodation and convergence responses.

A transient overshoot of the accommodative response to a step change in stimulus has been observed in previous reports, albeit each with data on one subject [3, 4].

The present study aims to explore further dynamic accommodative responses when viewing a stereoscopic display. In general, the measurement of the dynamic accommodation is affected by convergence due to induced misalignment of the visual axes. To solve this problem a video refraction unit was used which was designed to compensate for misalignment and enable simultaneous measurement under natural conditions of accommodation, convergence and pupillary responses. A previous pilot study [5] demonstrated that the accommodation measurement error was less than 0.2 $\mathrm{D}$ when the deviation from the primary visual axis was within 5 degrees .

\section{Methods}

\subsection{Stimulus}

The stereoscopic liquid crystal display (LL-151D, Sharp, Osaka” Japan) was placed at a distance of $33 \mathrm{~cm}$ from the viewer's eye. A resolution of the display was $1024 \times$ 768 pixels. The display has a parallax barrier (a series of $60 \mu \mathrm{m}$ width slits) to generate images to the two eyes separately. Usually, parallax barrier systems designed to allow the two eyes to observe different images have the parallax barrier set in front of the screen. The present LCD has, however, a different type of parallax barrier, which is composed of an additional LCD panel which is set between the main LCD screen and the illumination generating system. Creating a parallax barrier using an 
LCD allows it to be removed for non-stereoscopic use. The size of the main LCD panel is 307 x $230 \mathrm{~mm}$. Each pixel is composed from red, green and blue (RGB) subpixels horizontally.

A high contrast ( 95\%) black Maltese cross was displayed against a white background $\left(34 \mathrm{~cd} / \mathrm{m}^{2}\right)$. The angular subtense of the Maltese cross was 6.11 degrees in both width and height. The Maltese cross was presented with three levels of Gaussian blur, which were achieved by retouch software (Adobe Photoshop, Adobe System Incorporated, San Jose, CA, USA). The Gaussian blur was produced by convolution of a target pattern with a Gaussian function to produce 0 (no blur), 16, and 32 minutes of arc represented by the radius of half width at half height of the Gaussian form.

Two conditions for target motion were used, both controlled by animation software (Flash MX, Macromedia, San Francisco, CA, USA). The first involved the target being moved repeatedly in a step-wise manner between a no-disparity image condition at $50 \mathrm{~cm}$ and pop-up stereoscopic image condition at $33 \mathrm{~cm}$ drawn on a 50 cm screen with stereoscopic mode (i.e. active parallax barrier) using a setup as shown in Figure 1. The disparity of the target changed as the apparent distance changed from $50 \mathrm{~cm}$ (black solid line with arrow in Figure 1) to $33 \mathrm{~cm}$ (green solid lines) while the accommodative stimulus was maintained at the constant screen surface distance. The condition is denoted as 2MA-2D $<=>3$ MA-2D (MA: meter angle, D: diopter). The second condition was such that the display was presented in non-stereoscopic mode, that is the parallax barrier was not used for comparison with the first condition. In this condition the target was presented through the semi-combiner at $33 \mathrm{~cm}$ (black broken line with arrow in Figure 1) or $50 \mathrm{~cm}$ (black solid line with arrow) from viewer's eyes. The condition is denoted as $2 \mathrm{MA}-2 \mathrm{D}<=>3 \mathrm{MA}-3 \mathrm{D}$. The target was presented for 5 seconds in each condition. A photocell was incorporated into the display and used to monitor and time-lock the switch between stimulus conditions via a PC (Windows XP machine, self-built).

$==+==+=$

Figure 1 will be inserted around here

$==+=+=$ 


\subsection{Measurements}

Accommodation and convergence were measured dynamically at the rate of 30 $\mathrm{Hz}$ using a modified commercially-available video refraction unit (PR-1000, TOPCON, Tokyo, Japan). Image analysis was carried out using virtual instrument software (LabView 7.0 with vision development tool 7.0, National instruments, Austin, Texas USA). Dynamic responses were measured real-time at a rate of $30 \mathrm{~Hz}$ by extending previouly reported methods [3] which analysed stored video images, using a photorefraction technique [5].

\subsection{Subjects}

Initially potential subjects were shown 2-3 cycles to determine whether fusion was possible. Those subjects for whom fusion was impossible without training were excluded from the study.

Six male subjects (age range from 22 to 25 years, mean $=23.5$ ) and one female subject (age 22 years) participated. All had normal vision and oculomotor functions including accommodative amplitude. Fusional reserve was not measured. Instead, fusion ability to the present set-up was a criteria to take part in the study.

Only one subject (AY) wore ophthalmic lenses (around -4 D in both eyes), another (YO) wore contact lenses and the rest were emmetropic.

\subsection{Procedure}

In the experimental trials, 6 or 7 steps were recorded in each subject in each condition to minimise prediction and fatigue effects. The latter 5 traces were analysed to allow the subject time to adapt to the experimental conditions. A period of 1 minute was given between trials to also minimise prediction and fatigue effects.

Subjects were instructed to try to fuse the stereoscopic images while maintaining clarity. The average blink rate is once every 8 seconds, so they were instructed to blink just after near-to-far motion to not affect the analysed responses while maintaining natural blinking.

The analysis of static accommodative responses matched that used in a previous report [2]. A static accommodative response, which refers to a stable response after transient response, was defined as the difference between the average during 2.5 seconds before the onset of the stimulus and average from 2.5 seconds to 5.0 seconds 
after onset of the stimulus. In addition, the overshoot peak responses of the dynamic accommodative responses were calculated as the difference between the peak value and the averaged value for the 2.5 seconds before the onset of the stimulus. The analysis of the static convergence measurements was performed similarly.

\section{Results}

Various waveforms were observed with regard to the dynamic accommodative responses to stereoscopic conflicting stimulus. A transient overshoot was observed in four subjects and oscillation was observed in one subject. A further two subjects had a stable accommodative response without overshoot and oscillation. Figure 2 shows typical examples of these responses for the conflicting condition. Each waveform is the average of 5 trials except for the subject showing oscillation (Figure 2b) which would have been masked by this presentation format.

Figure 2a shows the typical overshoot response. The dynamic accommodative overshoot was observed in the conflicting condition, but was not observed either in the non-conflicting condition or in the convergence data. The oscillation of accommodation and convergence was only evident in one subject when the target had no blur as shown in Figure 2b. When the target was blurred by 16 or 32 min arc, an overshoot of accommodative response was observed instead of an oscillating trace; convergence showed a stable response. The oscillation present in the convergence responses indicates that the subject could not fuse the 3MA-2D target under no-blur conditions. Figure 2c shows the typical response of those subjects who showed no accommodative overshoot or oscillatory response. There are no special features of note in either accommodation or convergence responses.

In the non-conflicting condition, only one subject (AY) continued to show an overshoot of accommodative response as in the conflicting condition whilst the other subjects showed a smooth response to the range of targets presented (Figure 3a-3c). The peak and static responses in accommodation for all subjects are shown in Figure 4 and convergence in Figure 5. In Figures 4 and 5, panels a, b, c and d show the results for the subjects who had an overshoot of accommodative response. Panel e, demonstrates how subject TN accommodative and convergence responses oscillated and panels $\mathrm{f}$ and $\mathrm{g}$ demonstrates the response of the two subjects showing a smooth 
oculomotor response. The dotted lines show the peak responses in accommodation and convergence for the conflicting (2MA-2D => 3MA-2D) and non-conflicting (2MA-2D => 3MA-3D) conditions whereas the solid lines show the static responses for the conflicting and non-conflicting conditions. As shown in Figure 4a-4e, the static accommodation responses for the conflicting conditions are greater with increased target blur in those subjects who showed accommodative overshoot or oscillation. An equivalent result was reported in a previous study [3]. The accommodative responses under the conflict condition were most marked for the 32 minutes of arc blur, modest for 16 minutes of arc blur and smallest for the non-blurred target.

Conversely, there is no clear trend in static accommodative responses for the conflicting condition in the two subjects who showed a smooth accommodative response to the change in image vergence, here the responses were relatively constant with respect to target blur.

The static responses for the non-conflicting 3MA-3D accommodation condition are relatively constant in all subjects with respect to level of target blur. Static accommodative responses for the non-conflicting condition are larger than those for the conflicting 3MA-2D condition in five subjects, but smaller in one subject, KS, and comparable in one subject, AY.

$==+===$

Figures 2-5 will be inserted around here

$======$

\section{Discussion}

Dynamic oculomotor responses during the viewing stereoscopic image is characterized by substantial variation, such as overshoot, oscillation and stable responses. Usually accommodation responses are cross-linked with convergence, but they can occur independently from each other; as shown in the present results where overshoot waveforms were observed only in accommodative responses. In contrast, accommodative oscillation is invariably accompanied by the convergence oscillation. Oscillation responses are generally considered to represent difficulty in fusing 
stereoscopic images as described in our previous study [3]. Fusion difficulty depends, of course, on the amount of disparity and on individual capability. The present study shows that image resolution also affects fusion difficulty with one subject showing oscillation in responses only when the image had higher spatial frequency components. Hence improving image quality in terms of spatial characteristics will not necessarily optimise the quality of the stereoscopic image.

There may be two possible explanations for the accommodative overshoot observed when the stereoscopic image is used for accommodative stimulus.

Accommodative responses for one stable subject are shown in Figure 2c. Close observation of the response patterns indicates a slow and constant increase of accommodative response that occurs typically over 1 second and subsequent to the initial fast components. This observation suggests that the fast initial accommodative response to step stimuli is probably small for this group. In contrast an overshoot in accommodation response indicates a large initial fast response. The first hypothesis concerns inter-individual variation in $\mathrm{CA} / \mathrm{C}$ ratio, which for a given amount of convergence is defined as the ratio of convergence stimulated accommodation to convergence and is an index of the strength of convergence-driven accommodation. The fast initial component of accommodative response is derived from a convergence stimulus and subsequent convergence response. Thus the $\mathrm{CA} / \mathrm{C}$ ratio determines the initial accommodative response and hence the variation in waveform of the accommodative response may be attributable to inter-individual differences of $\mathrm{CA} / \mathrm{C}$ ratio [7]. The amount of overshoot varied along with target blur in subject $\mathrm{MM}$ as shown in Figure 2a. Clearly the initial fast accommodative response has been affected. However, if the initial fast component is determined by the CA/C ratio it should be independent of target blur and this finding is difficulty to account. Further, Figure 3a (non-conflicting stimuli) shows for subject MM that the initial accommodative responses are maintained for the non-conflicting 3MA-3D stimuli. From these data it can therefore be deduced that accommodative overshoot is unlikely to be attributable to a higher $\mathrm{CA} / \mathrm{C}$ ratio.

A second theory is as follows. During the blur is large, the amount of blur is not used to control accommodation at the initial fast response of accommodation to step stimuli, and go/stop control is achieved. After the stop judgment when the blur becomes small, slow and fine control system follows to minimise blur [8, 9]. This relationship explains why over-accommodation occurs with stimulus conflict but is 
stable for non-conflicting stimuli. During convergence-driven accommodation, the accommodation control system is unable to respond although blur is increasing. Thus overshoot response in accommodation to stereoscopic image can be explained. However, the data do not fully support the above hypothesis as there are individuals who exhibit stable responses when stimuli are in conflict. The stable subject's accommodation response (figure 2c) drifted towards distance rather than near once the initial fast phase was completed. In this case it is easier to consider that the initial first phase was too small in these subjects, probably due to the low $\mathrm{CA} / \mathrm{C}$ ratio and hence more consistent with the first rather than with the second theory.

In any event we envisage that additional data will help in the construction of an extended interactive model of accommodation and convergence control system [10].

The response traces were reviewed for prediction effects but, within the 5 stepped responses lasting less than 1 minute, none were observed as is confirmed by the consistent response and accommodative overshoot in many of the subjects. The finding that subjects in the stable group did not show overshoot responses despite repeated trials indicates that task learning and response expectancy did not constitute a significant role in determining the accommodation response.

Static accommodative responses to stereoscopic stimuli depend on the spatial frequency components of the target such that accommodation responses increase with increased target blur [2]. The static accommodative response is determined by the balance of defocus-driven accommodation and convergence-driven accommodation. If the spatial frequency profile of a target contains higher spatial frequencies, the tolerance of defocus becomes smaller, defocus-driven accommodation becomes stronger and hence accommodative responses diminish for targets without blur. Support for this relationship is provided in this study with reference to data for the overshoot subjects and non-oscillation data evident for the oscillation subject (see Figure 4a to 4e) although data for two stable subjects were equivocal (see Figures $4 \mathrm{f}$ and $4 \mathrm{~g})$. Even though the spatial component dependency of the accommodative static response does not include variation in $\mathrm{CA} / \mathrm{C}$ ratio, it may be influenced by intersubject variation in $\mathrm{CA} / \mathrm{C}$.

Under the non-conflicting condition, where the accommodative stimuli and convergence stimuli are matched, static accommodative responses are consistent with blur (see Figure 5). Although defocus-driven accommodation may vary with target blur it is evident that static accommodation was not affected by the target blur. This 
means that convergence-driven accommodation predominates over defocus-driven accommodation unless the large error in the accommodation control system is not detected.

Sometimes an initial erroneous direction of response was observed especially in the accommodation response (Figure 2a near-to-far and 2c far-to-near). Although it was frequent in one subject MM, in one direction (near-to-far), and for one condition (conflicted stereoscopic stimulus), few erroneous responses were found in general. Lack of the psychological cues for depth perception such as size change may contribute to this phenomenon [11]. However, the fact that the convergence response showed no instances of erroneous initial direction of response cannot be explained by the theory that the initial accommodative responses are driven by the convergence responses. When an erroneous direction in accommodative response occurred, accommodation and convergence responses were in conflict. Thus the theory shown here is not perfect and further study is needed to complete the model which can explain the present data fully.

This work is supported by the Japanese Ministry of Education, Science, Sports and Culture, Grant-in-Aid for Scientific Research (B), 16300034, 2004-2006. 


\section{References}

[1] A. Wilkins: Visual stress, Oxford Science Publications, Oxford (1995)

[2] Y. Okada, K. Ukai, J.S. Wolffsohn, et al., Vision Res., 46, 475 (2006).

[3] K. Ukai and Y. Kato, Ophthal. Physiol. Opt., 22, 385 (2002).

[4] T. Inoue and H. Ohzu, App. Opt., 36, 4509 (1997)

[5] H. C. Howland and B. Howland, J. Opt. Soc. Am., 64, 240 (1974)

[6] J. Katori , Y. Komachi, K. Ukai, et al, Ganka-Rinsho-Iho, 86, 87 (1992)

[7] M. Rosenfield, K. J. Ciuffreda and H. W. Chen: Ophthal. Physiol. Opt., 15, 451 (1995)

[8] T. Yamada and K. Ukai, Ophthal. Physiol. Opt., 17, 55 (1997)

[9] S. R. Bharadwaj and C. M. Schor, Vision Res., 46, 1959 (2006)

[10] C. M. Schor: Optom. Vis. Sci., 69, 258 (1992)

[11] L. Stark and Y. Takahashi: IEEE Trans. Biomed. Eng., 12, 138 (1965) 
Figure Captions

Figure 1 Schematic drawing of the apparatus. Two areas of LCD were used for the 3MA-3D and 2MA-2D targets. The 3MA-2D target was generated by showing stereoscopic images in the area of 2MA-2D.

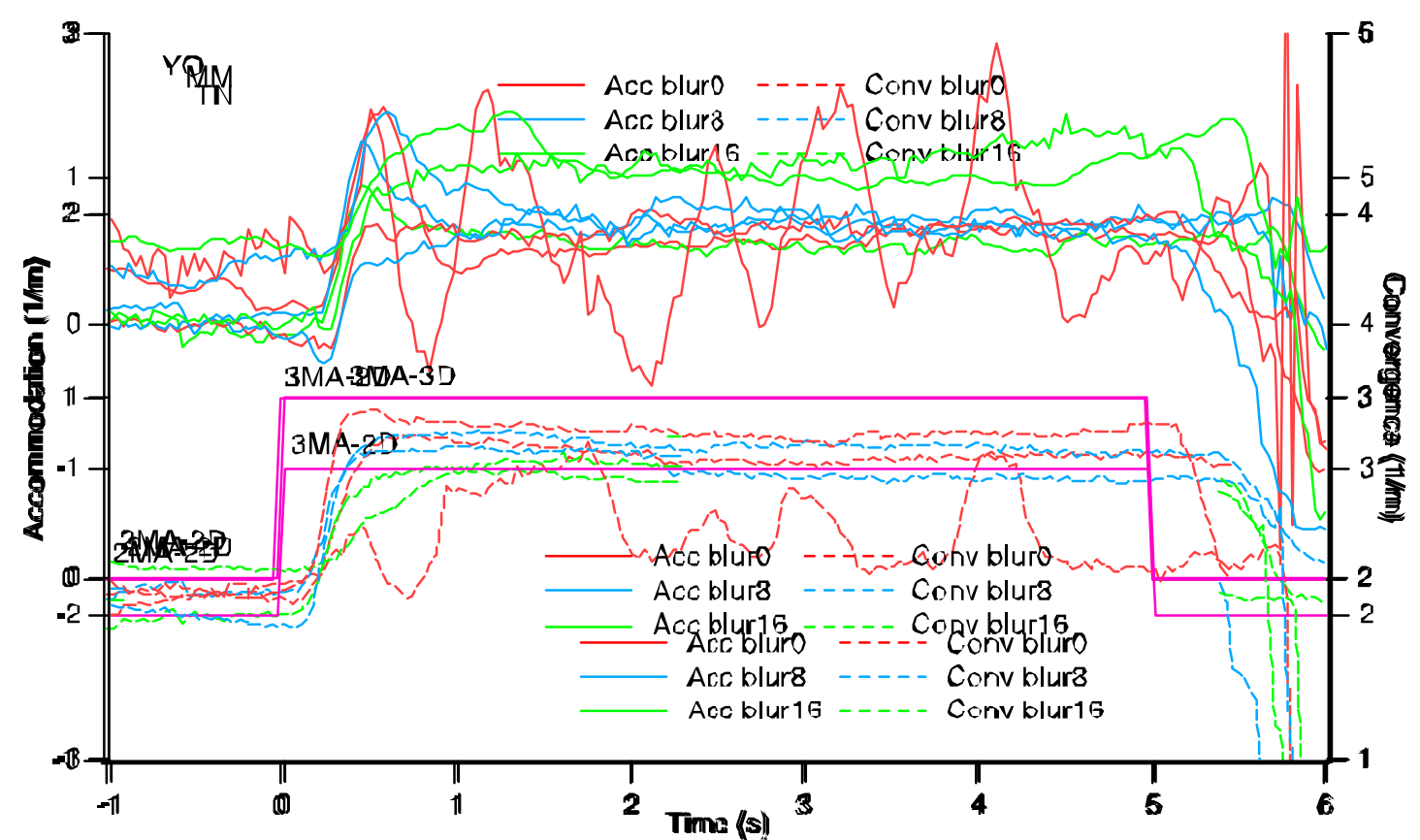

Figure 2: Typical accommodative and convergence responses for each group in conflicting stereoscopic condition. Each waveform (apart from 2b) is an average of 5 trials for a single subject. Solid lines: Accommodation response (see left scale), Broken line: Convergence response (see right scale). Red: no-blur, Green: Target with blur of 16 min arc, Blue: Responses to a target with 32 minn convergence were observed when the target blur was 16 and 32 min arc. (c) Typical responses of the stable subjects.

Figure 3: Typical accommodative and convergence responses for non-conflicting condition. Key: see Figure 2.

Figure 4: Accommodative static responses and overshoot peak responses in all subjects. Red squares with solid lines: 3MA-3D. Blue squares with solid lines: 3MA2D. Blue diamonds with broken lines: overshoot peak responses of accommodation to 3MA-2D stimulus. (a)-(d) Subjects MM, AY, KS and MT in the overshoot group. (e) Subject TN who had oscillation. Static and overshoot peak responses to 3MA-2D stimulus with no target blur could not be measured due to oscillation responses. (f), (g) Subjects YO and HS in the stable group. Peak responses to 3MA-3D in a, c, and d are 
not measured because only subject AV had an overshoot to the non-conflicting stimulus (3MA-3D).

Figure 5: Amount of convergence static responses. Red: non-conflicting. Blue: conflicting. (a)-(g) Refer to Figure 4. 
Figure 1

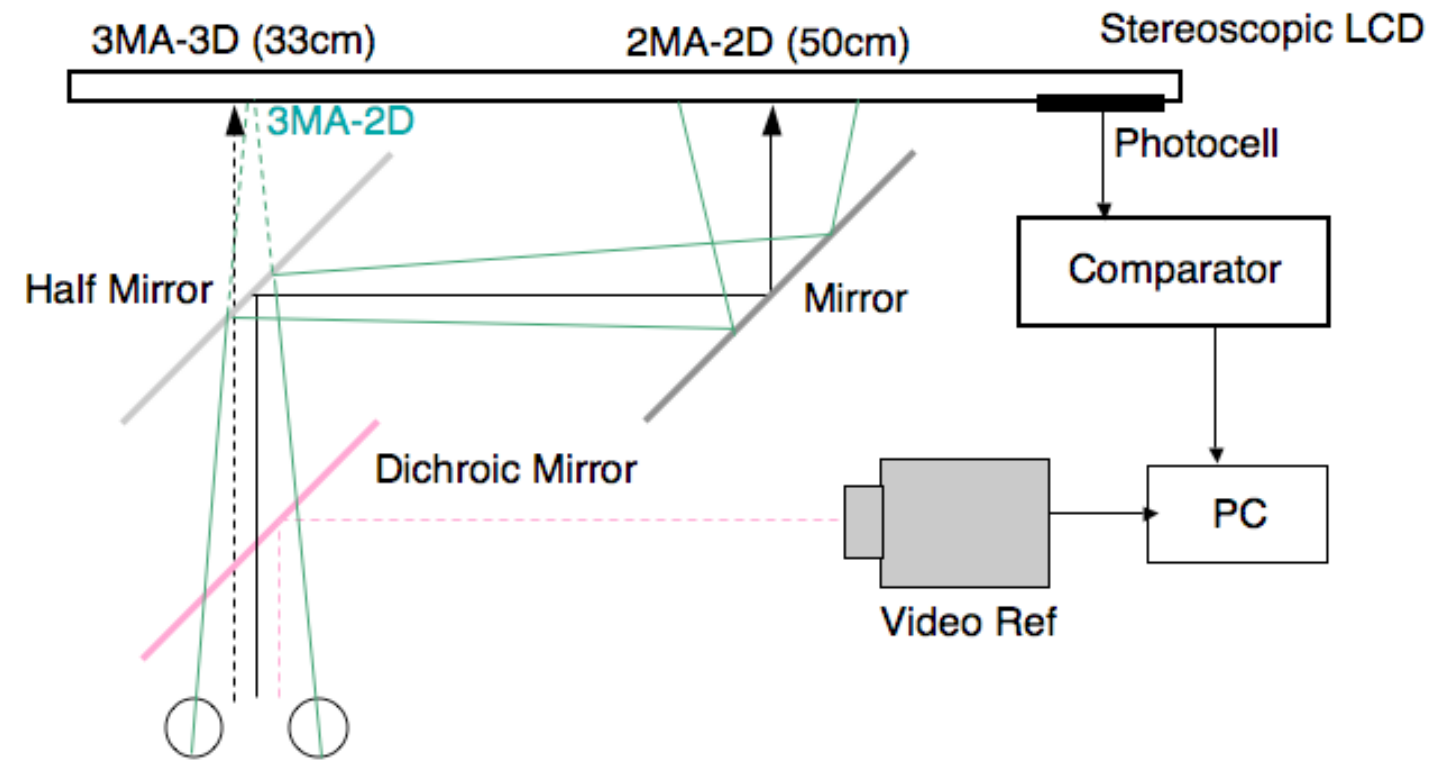


Figure 2c

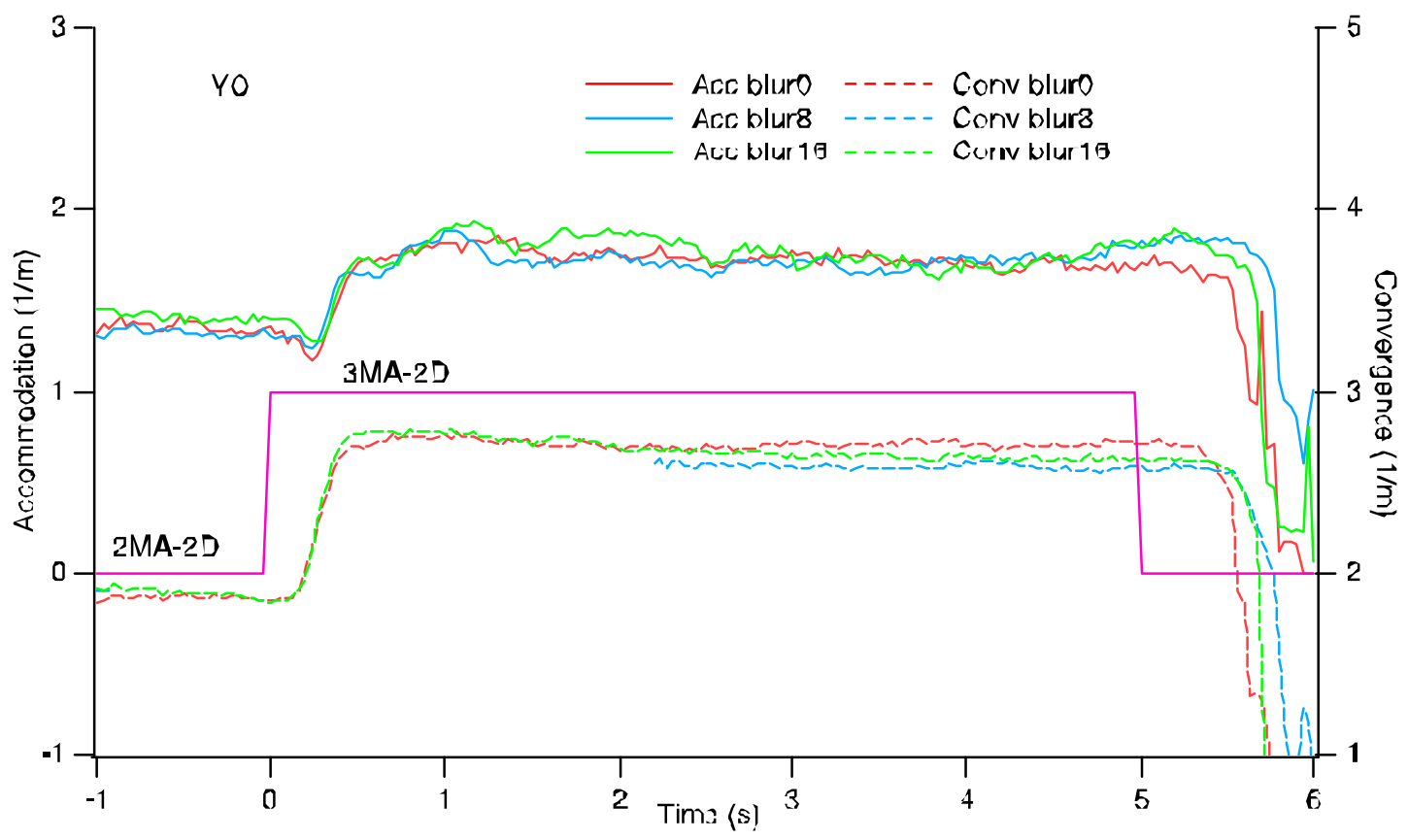


Figure3a

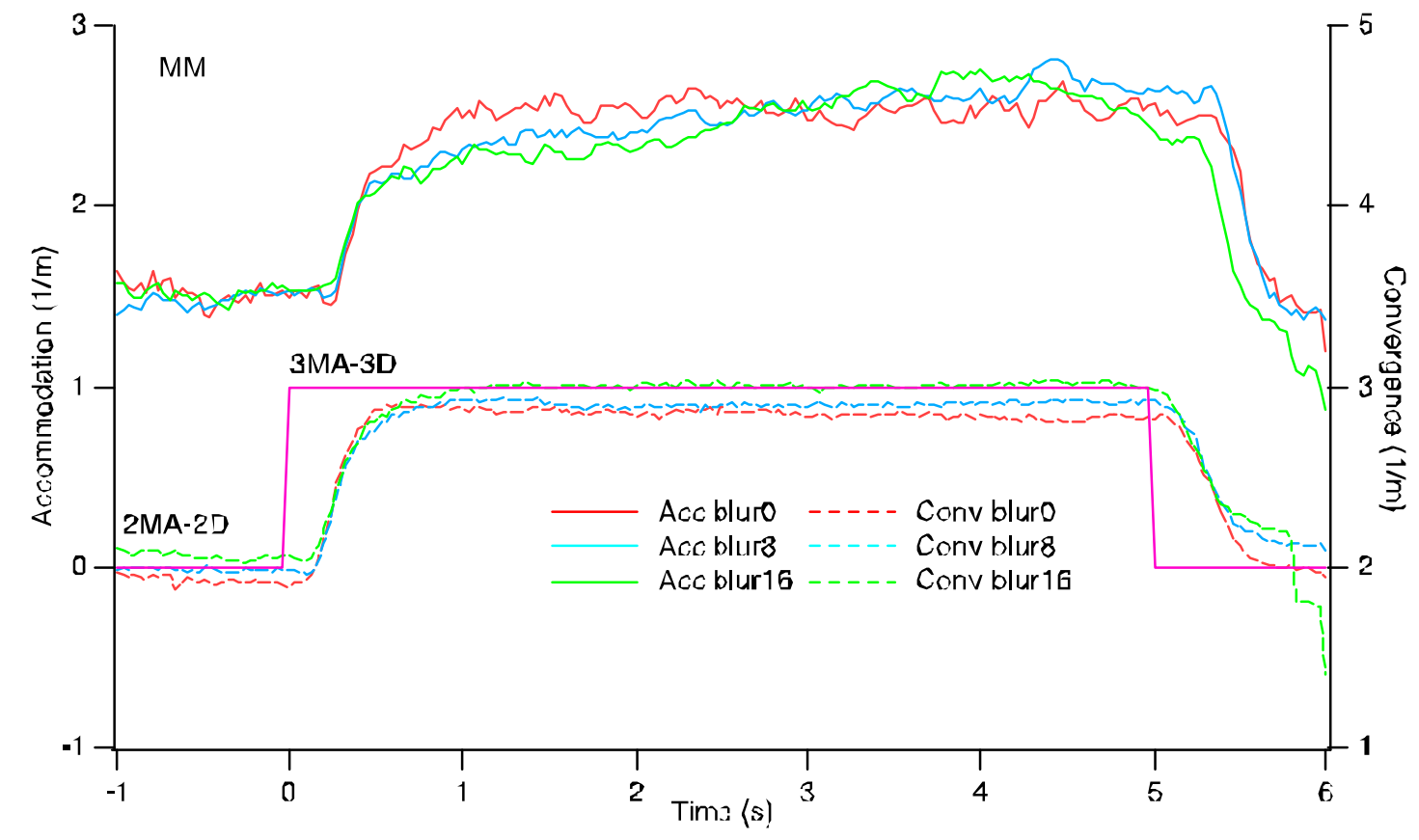


Fig 3b

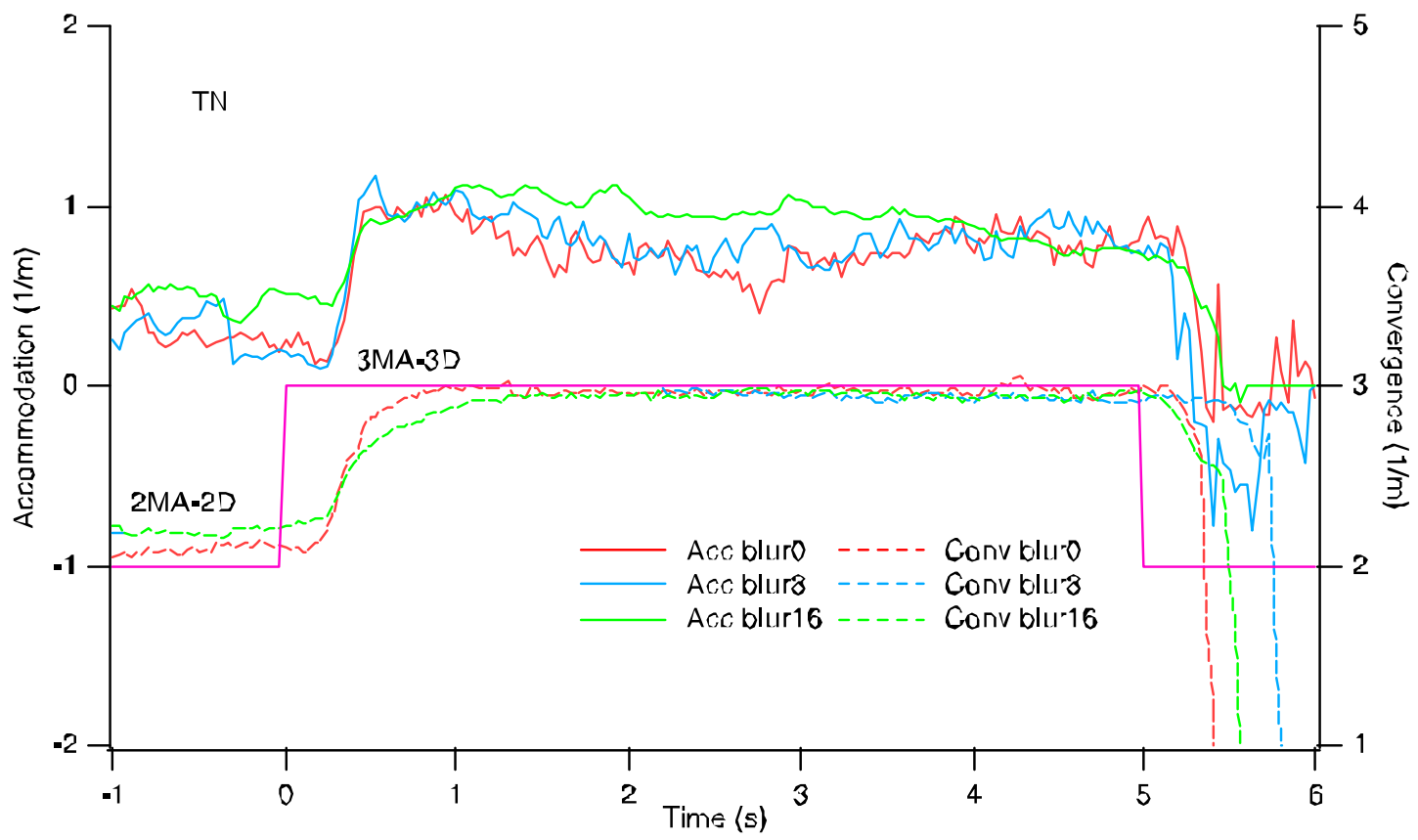

